\title{
Open projections do not form a right residuated lattice
}

\author{
David Kruml* \\ Masaryk University, Brno, Czech Republic \\ kruml@math.muni.cz
}

A lattice $L$ with operations $\odot, \multimap$ is called right residuated if

$$
x \odot y \leq z \Leftrightarrow x \leq y \multimap z
$$

for all $x, y, z \in L$. (So we do not require the dual arrow nor the associativity of $\odot$.) In other words, the actions $-\odot y, y \multimap-: L \rightarrow L$, for any fixed $y \in L$, provide a Galois connection on $L$.

The Galois connection is also completely determined by the partial isomorphism between fixpoints of closure mapping $y \multimap(-\odot y)$ and coclosure mapping $(y \multimap-) \odot y$. Thus we can restrict $\odot, \multimap$ to partial operations $\cdot, \rightarrow$ where $x \cdot y$ is defined if $x=y \multimap(x \odot y)$ and $y \rightarrow z$ is defined if $z=(y \multimap z) \odot y$. The partial isomorphism provides an equivalence

$$
x \cdot y=z \Leftrightarrow x=y \rightarrow z
$$

(We use a similar idea as in [6] but there the partial residuation law use standard inequalities. Cf. this also with [3].)

Conversely, whenever we have such a partial right residuated lattice, i.e. $L$ satisfies $(*)$, for each $y$ the --compatible elements define a closure $x \mapsto \hat{x}$ and the $\rightarrow$-compatible elements define a coclosure $z \mapsto \check{z}$, then by putting $x \odot y=\hat{x} \cdot y$ and $y \multimap z=y \rightarrow \check{z}$ we get a total right residuated structure on $L$.

1 Example. (1) Every orthomodular lattice with Sasaki operations

$$
x \odot y=\left(x \vee y^{\perp}\right) \wedge y, \quad y \multimap z=y^{\perp} \vee(y \wedge z)
$$

is right residuated. The partial product $x \cdot y$ is defined for $x \geq y^{\perp}$, and $y \rightarrow z$ is defined for $z \leq y$. Thus - is a restriction of the meet operation on compatible elements.

(2) In the MV-chain $[0,1]$ with $x \odot y=\max \{0, x+y-1\}$ the partial product is defined also for $x \geq y^{\perp}=1-y$, i.e. whenever $x+y-1 \geq 0$.

Note that in orthomodular lattices $\odot$ can be recovered from the meet defined on all pairs of compatible elements and such a partial operation also provides the closure and coclosure mappings. In that sense $\cdot$ is the minimal such generating partial operation.

The partial operations $\cdot, \rightarrow$ may have better algebraical properties than the total operations $\odot, \multimap$, e.g. in orthomodular lattices $\cdot$ is associative and commutative while $\odot$ is not. Sometimes it can be useful to study right residuated (or even residuated) structures by the partial operations [2].

In [4] and [1] the authors considered the lattice of closed right ideals (or equivalently so called open projections) as a spectrum of non-commutative $\mathrm{C}^{*}$-algebra. The embedding of a $\mathrm{C}^{*}$ algebra to an enveloping $\mathrm{W}^{*}$-algebra provides a representation of the lattice to an orthomodular

*Supported by the project "Algebraic Methods in Quantum Logic" by ESF, No. CZ.1.07/2.3.00/20.0051. 
lattice. In commutative case, the lattice is a just frame (the topology given by Gelfand-Naimark duality) and so a residuated lattice (as a complete Heyting algebra).

The lattice of open projections itself is not sufficient to recover the $\mathrm{C}^{*}$-algebra but it is sufficient when it is equipped with a partial meet operation on compatible elements [5]. It is a natural question whether such a partial operation extends to a total right residuated operation. Using the above ideas I will show an example of $\mathrm{C}^{*}$-algebra where there is no such extension and which disproves a conjecture in [5] that a product of open projections is open.

Recall from [4] that a projection $p \in A^{* *}$ (here $A^{* *}$ is the enveloping $\mathrm{W}^{*}$-algebra of $\mathrm{C}^{*}$ algebra $A$ ) is called open if it is a support of some $a \in A$, i.e. the smallest projection such that $a p=a$.

2 Example. Let $A$ be a $\mathrm{C}^{*}$-algebra which elements are norm-convergent sequences of $2 \times 2$ matrices $a_{n}$ together with their limits, denoted by $a_{\infty}$, i.e. $\left(\left(a_{n}\right)_{n \in \mathbb{N}}, a_{\infty}\right) \in A$ iff $a_{n} \rightarrow a_{\infty}$. The enveloping $\mathrm{W}^{*}$-algebra $A^{* *}$ simply contains all sequences and the element $a_{\infty}$ need not be a limit of $a_{n}$. The corresponding orthomodular lattice is a countable power of the orthomodular lattice of subspaces of $\mathbb{C}^{2}$ and the Sasaki operations are calculated componentwise.

Sequence $a_{n}=\left(\begin{array}{cc}1 & 0 \\ 0 & 1 / n\end{array}\right), a_{\infty}=\left(\begin{array}{ll}1 & 0 \\ 0 & 0\end{array}\right)$ belongs to $A$ and since all matrices $a_{n}$ are regular, its support is $p_{n}=\left(\begin{array}{ll}1 & 0 \\ 0 & 1\end{array}\right), p_{\infty}=\left(\begin{array}{ll}1 & 0 \\ 0 & 0\end{array}\right)$. Constant sequence $q_{n}=q_{\infty}=\frac{1}{2}\left(\begin{array}{ll}1 & 1 \\ 1 & 1\end{array}\right)$ is already a projection in $A$.

Let us consider the projections $p=\left(p_{n}\right), q=\left(q_{n}\right)$ as elements of $A^{* *}$. Complementary projection $\neg p$ is given by $\neg p_{n}=\left(\begin{array}{ll}0 & 0 \\ 0 & 0\end{array}\right), \neg p_{\infty}=\left(\begin{array}{ll}0 & 0 \\ 0 & 1\end{array}\right)$ and clearly is not open. But we also have $(q \odot p)_{n}=((\neg p \vee q) \wedge p)_{n}=\left(\left(\begin{array}{ll}0 & 0 \\ 0 & 0\end{array}\right) \vee \frac{1}{2}\left(\begin{array}{ll}1 & 1 \\ 1 & 1\end{array}\right)\right) \wedge\left(\begin{array}{ll}1 & 0 \\ 0 & 1\end{array}\right)=\frac{1}{2}\left(\begin{array}{ll}1 & 1 \\ 1 & 1\end{array}\right)$ while $(q \odot p)_{\infty}=\left(\left(\begin{array}{ll}0 & 0 \\ 0 & 1\end{array}\right) \vee \frac{1}{2}\left(\begin{array}{ll}1 & 1 \\ 1 & 1\end{array}\right)\right) \wedge\left(\begin{array}{ll}1 & 0 \\ 0 & 0\end{array}\right)=$ $\left(\begin{array}{ll}1 & 0 \\ 0 & 0\end{array}\right)$, thus $(q \odot p)$ is not open too.

Finally, let $r^{m}, s^{m}$ be collections of sequences for each $m \in \mathbb{N}$ given by $r_{n}^{m}=s_{n}^{m}=\frac{1}{2}\left(\begin{array}{ll}1 & 1 \\ 1 & 1\end{array}\right)$ for $n<m, r_{n}^{m}=s_{n}^{m}=\left(\begin{array}{ll}1 & 0 \\ 0 & 1\end{array}\right)$ for $n \geq m$, and $r_{\infty}^{m}=\left(\begin{array}{ll}1 & 0 \\ 0 & 0\end{array}\right)$ while $s_{\infty}^{m}=\left(\begin{array}{ll}0 & 0 \\ 0 & 0\end{array}\right)$.

Each projection $r^{m}$ or $s^{m}$ is open. But the componentwise intersection of $r^{m}$ is not open because the limit component is $\left(\begin{array}{ll}1 & 0 \\ 0 & 0\end{array}\right)$ while all other components are $\frac{1}{2}\left(\begin{array}{ll}1 & 1 \\ 1 & 1\end{array}\right)$. Thus $\bigwedge r^{m}$ (calculated in $A$, i.e. the interior of the discussed intersection) is given by $\left(\bigwedge r^{m}\right)_{n}=\frac{1}{2}\left(\begin{array}{ll}1 & 1 \\ 1 & 1\end{array}\right),\left(\bigwedge r^{m}\right)_{\infty}=$ $\left(\begin{array}{ll}0 & 0 \\ 0 & 0\end{array}\right)$, hence $\bigwedge r^{m}=\bigwedge s^{m}$.

Since $r^{m}, s^{m} \leq p$ for each $m$, "compatible arrows" $p \rightarrow r^{m}, p \rightarrow s^{m}$ are defined and $(p \rightarrow$ $\left.r^{m}\right)_{n}=\left(p \rightarrow s^{m}\right)_{n}=\frac{1}{2}\left(\begin{array}{ll}1 & 1 \\ 1 & 1\end{array}\right)$ for $n<m,\left(p \rightarrow r^{m}\right)_{n}=\left(p \rightarrow s^{m}\right)_{n}=\left(\begin{array}{ll}1 & 0 \\ 0 & 1\end{array}\right)$ for $n \geq m$, and $\left(p \rightarrow r^{m}\right)_{\infty}=\left(\begin{array}{ll}1 & 0 \\ 0 & 1\end{array}\right)$ while $\left(p \rightarrow s^{m}\right)_{\infty}=\left(\begin{array}{ll}0 & 0 \\ 0 & 1\end{array}\right)$. Notice that all $p \rightarrow r^{m}, p \rightarrow s^{m}$ are open and $\left(\bigwedge p \rightarrow r^{m}\right)_{n}=\left(\bigwedge p \rightarrow s^{m}\right)_{n}=\frac{1}{2}\left(\begin{array}{ll}1 & 1 \\ 1 & 1\end{array}\right),\left(\bigwedge p \rightarrow r^{m}\right)_{\infty}=\frac{1}{2}\left(\begin{array}{ll}1 & 1 \\ 1 & 1\end{array}\right)$ while $\left(\bigwedge p \rightarrow s^{m}\right)_{\infty}=\left(\begin{array}{ll}0 & 0 \\ 0 & 0\end{array}\right)$. Thus $\bigwedge p \rightarrow r^{m} \neq \bigwedge p \rightarrow s^{m}$ and hence $p \multimap \bigwedge r^{m}$ can not exist because $p \multimap-$ should preserve all infima.

3 Corollary. Product of open projections need not be open.

4 Corollary. The partial monoid structure on compatible open projections need not extend to a right residuated structure on all open projections.

\section{References}

[1] Akemann, C. A. Left ideal structure of C*-algebras. Journal of Functional Analysis 6 (1970), 305-317.

[2] Chajda, I., Halaš, R., And Kühr, J. Every effect algebra can be made into total algebra. Algebra Universalis 61(2) (2012), 139-150.

[3] Chajda, I., And KrňÁvek, J. Skew residuated lattices. Fuzzy sets and systems (2013). in press.

[4] Giles, R., And Kummer, H. A non-commutative generalization of topology. Indiana University Mathematics Journal 21(1) (1971), 91-102. 
[5] Kruml, D. On definition of skew frames. International Journal of Theoretical Physics 49 (2010), 3153-3157.

[6] Zhou, X., And Li, Q. Partial residuated structures and quantum structures. Soft Computing 12 (2008), 1219-1227. 